\title{
Erratum to: Action recognition with spatio-temporal augmented descriptor and fusion method
}

\author{
Lijun $\mathrm{Li}^{1} \cdot$ Shuling Dai ${ }^{1}$
}

Published online: 7 September 2016

(C) Springer Science+Business Media New York 2016

\section{Erratum to: Multimed Tools Appl}

DOI 10.1007/s11042-016-3789-0

The published version of this article unfortunately contained an error. In the published paper, the Equation (7) was incorrect. It should be written as following:

$$
\begin{aligned}
K\left(x_{i}, x_{j}\right)= & \sqrt{d_{1}} R_{i}^{1^{T}} \cdot \sqrt{d_{1}} R_{j}^{1}+\ldots+\sqrt{d_{m}} R_{i}^{m^{T}} \cdot \sqrt{d_{m}} R_{j}^{m} \\
= & d_{1} K_{1}\left(R_{i}^{1}, R_{j}^{1}\right)+\ldots+d_{m} K_{m}\left(R_{i}^{m}, R_{j}^{m}\right)=\sum_{k=1}^{m} d_{k} K_{k} \\
& \sum_{k=1}^{m} d_{k}=1
\end{aligned}
$$

The online version of the original article can be found at http://dx.doi.org/10.1007/s11042-016-3789-0.

Lijun Li

lilijun1990@buaa.edu.cn

Shuling Dai

sldai@yeah.net

1 State Key Laboratory of Virtual Reality Technology and Systems, Beihang University, Beijing, China 